Kong. Res. J. 3(1) : 59-64, 2016

ISSN 2349-2694

Kongunadu Arts and Science College, Coimbatore.

\title{
EXPLORATION OF ORNAMENTAL FLORAS IN THE CAMPUS OF S.T. HINDU COLLEGE, NAGERCOIL, KANYAKIMARI DISTRICT, TAMILNADU, INDIA
}

\author{
Mary Kensa, $\mathbf{V}^{1 *}$., G. Sahaya Anthony Xavier ${ }^{2}$, A. Asha ${ }^{1}$, B. Brintha ${ }^{1}$, M. Pechiammal ${ }^{1}$ and B. Praveena ${ }^{1}$ \\ ${ }^{1}$ Department of Botany and Research Centre, S.T. Hindu College, Nagercoil. \\ ${ }^{2}$ Department of Botany, St.Xavier's College, Palayamkottai. \\ *E.mail: surejkensa@gmail.com
}

\begin{abstract}
Most of the present day flowers have come from the wild progenitors, a few of which still exist in natural habitat.Ornamental flowers are highly promising and unutilized resources having tremendous and prover economic importance.Ornamental plants accompany people, since their birth to death and they coexist with almost all happy events in life such birthday celebrations, weddings, carrier progress etc. In addition, they form our best partners in our everyday life in our flats, offices, different public spaces, parks, gardens and elsewhere.An extensive floristic survey was conducted during the year 2015. Taxonomic identification, photographic documentation and ornamental characterization of each species with potential for use on floral art were recorded. The methodology used is based on observation method for the determination of flora. All the specimens collected were identified with the help of recent literature.The field expeditions of study area gave interesting results concerning floristic diversity.
\end{abstract}

Keywords: Ornamental flowers, domestication, floristic diversity and methodology.

\section{INTRODUCTION}

Ornamental flowers are highly promising and unutilized resources having tremendous and prover economic importance (Jenomics, 2014). Ornamental plants accompany people, since their birth to death and they co-exist with almost all happy events in life such birthday celebrations, weddings, carrier progress etc. In addition, they form our best partners in our everyday life in our flats, offices, different public spaces, parks, gardens and elsewhere (Arora, 2013). They are an inseparable part of the culture of all nations and nationalities. This is the reason, why people since time immemorial have tried to improve or change flowers and other ornamental plants according to their imagination, dreams and practical aspects of planting.

\section{MATERIALS AND METHODS}

\subsection{Floristic Survey}

An extensive floristic survey was conducted during June - November 2015. Taxonomic identification, photographic documentation and ornamental characterization of each species with potential for use on floral art were recorded. The methodology used is based on observation method for the determination of flora. All the specimens collected were identified with the help of recent literature by local floras authored by Hooker,(19721987), Gamble and Fischer, (1915-1935)and Henry et al., 1989.

\section{RESULTS AND DISCUSSION}

The field expeditions of study area gave interesting results concerning floristic diversity. A total of 108 plant species are present in this study area (Table -I). Among the species, dicots were distributed in 36 families with 74 species, monocots in 13 families with 28 species; pteriophytes are in 4 families with 4 species and gymnosperms in 2 families with 2 species, 22 plant species are wild, 72 species are significantly ornamental and 14 plant species are wild or cultivated.When the percentage distributions are calculated it is found that $68.5 \%$ of dicots, $25.09 \%$ of monocots, $3.7 \%$ of pteridophytes, $1.8 \%$ of gymnosperms are present. This profiling indicated that the maximum ornamentals are dicots following monocots gymnosperms, pteridophytes and cacti. Among the total identified plants, Acanthaceae is dominant family 14.28. Whenthe percentage distribution is calculated it is found that $68.5 \%$ of dicots, $25.09 \%$ of monocots, $3.7 \%$ of pteridophytes, $1.8 \%$ of gymnosperms are present.

The classification of the ornamental flora based on the diversity of its utilization indicating that the maximum 4 of the plant can be used as aquatic ornamentals followed by 22 species as 
ornamentals trees, 24 species as ornamental shrubs, 27 species as ornamental herbs, 16 species as ornamental hedges and fencing, 4 species as ornamental succulents and cacti, 11 species as ornamental climbers 2. Regarding the habit wise distribution of identified plants, 22 species as trees, 48 species as herbs, 26 species as shrubs, 4 species as climbers and 8 species as succulents, 2 species can be used as ornamental foliage followed by 1 species

Table 1. Number of plants and its family, habits in the study area.

\begin{tabular}{|c|c|c|c|c|}
\hline S.No. & Plant Names & Family & Habits & $\begin{array}{c}\text { Wild/ } \\
\text { Cultivated }\end{array}$ \\
\hline $1 *$ & Adathoda vasica Nees. & Acanthaceae & $\mathrm{S}$ & W \\
\hline 2.* & Allamanda cathartica L. & Apocynaceae & $\mathrm{S}$ & $\mathrm{C}$ \\
\hline $3 .^{*}$ & Albizzi ajulibrissin L. & Mimosaceae & $\mathrm{T}$ & $\mathrm{C}$ \\
\hline $4 . * *$ & Aloe sps & Liliaceae & $\mathrm{Su}$ & $\mathrm{C}$ \\
\hline $5 . *$ & Albizzi alebbeck L. & Mimosaceae & $\mathrm{T}$ & $\mathrm{C}$ \\
\hline $6 .^{* *}$ & Alocasi amacrorhiza L. & Araceae & $\mathrm{H}$ & $\mathrm{C}$ \\
\hline 7.** & Anthurium sps & Araceae & $\mathrm{H}$ & $\mathrm{C}$ \\
\hline 8. & Araucaria sps & Araucariaceae & $\mathrm{T}$ & $\mathrm{C}$ \\
\hline 9.* & Aristalochia indica L. & Aristolocaceae & $\mathrm{H}$ & W \\
\hline $10 .^{* *}$ & Asparagus racemosus Willd. & Liliaceae & CL & $\mathrm{C}$ \\
\hline $11 *^{*}$ & Azadirachta indica A.Juss & Meliaceae & $\mathrm{T}$ & $\mathrm{C}$ \\
\hline $12 .^{*}$ & Balsam impatiens Royle. & Balsamnaceae & $\mathrm{H}$ & $\mathrm{C}$ \\
\hline $13 .^{*}$ & Barleria prionitis L. & Acanthaceae & $\mathrm{H}$ & W \\
\hline $14 .^{* *}$ & Beaureare curvata Lem. & Agavaceae & $\mathrm{S}$ & $\mathrm{C}$ \\
\hline $15 .^{*}$ & Begonia floccifera L. & Bignoniaceae & $\mathrm{H}$ & $\mathrm{C}$ \\
\hline $16 .^{*}$ & Bougainvillea spectabis Wild. & Nyctaginaceae & CL & $\mathrm{C}$ \\
\hline $17 .^{*}$ & Caesalpinia pulcherrima L. & Caesalpiniaceae & $\mathrm{S}$ & $\mathrm{C}$ \\
\hline $18 .^{* *}$ & Caladium bicolour Vent. & Araceae & $\mathrm{H}$ & $\mathrm{C}$ \\
\hline $19 .^{*}$ & Callistemon citrinus L. & Myrtaceae & $\mathrm{T}$ & $\mathrm{C}$ \\
\hline $20 *$ & Calotropis gigantean L. & Asclepiadaceae & $\mathrm{S}$ & W \\
\hline $21 * *$ & Caryota urens L. & Arecaceae & $\mathrm{T}$ & W \\
\hline $22 *$ & Cassia biflora L. & Caesalpiniaceae & $\mathrm{S}$ & $\mathrm{W}$ \\
\hline $23 .^{*}$ & Cassia fistula L. & Caesalpiniaceae & $\mathrm{T}$ & $\mathrm{C}$ \\
\hline $24 .^{* *}$ & Casuarina equisetifolia L. & Casurinaceae & $\mathrm{T}$ & $\mathrm{C}$ \\
\hline $25 .^{*}$ & Catharanthus roseus L. & Apocynaceae & $\mathrm{S}$ & $\mathrm{C}$ \\
\hline $26 .^{* *}$ & Chlorophytum cosmosum Thumb. & Liliaceae & $\mathrm{H}$ & $\mathrm{C}$ \\
\hline $27 .^{* *}$ & Cissus quadrangularis L. & Vitaceae & $\mathrm{Su}$ & $\mathrm{C}$ \\
\hline $28 .^{*}$ & Clerodendrum speciosum L. & Verbenaceae & $\mathrm{H}$ & $\mathrm{C}$ \\
\hline $29{ }^{*}$ & Clitoria ternatea $\mathrm{L}$. & Fabaceae & CL & $\mathrm{C}$ \\
\hline $30 .^{*}$ & Codiaeum variegatum L. & Euphorbiaceae & $\mathrm{S}$ & $\mathrm{C}$ \\
\hline $31 .^{*}$ & Coleus amboinicus Lour. & Lamiaceae & $\mathrm{H}$ & $\mathrm{C}$ \\
\hline $32 .^{*}$ & Coleus blumei Benth. & Lamiaceae & $\mathrm{H}$ & $\mathrm{C} / \mathrm{W}$ \\
\hline $33 .^{* *}$ & Commelina benghalensis L. & Commelinaceae & $\mathrm{H}$ & W \\
\hline $34 .^{* *}$ & Cordyline sps & Liliaceae & $\mathrm{S}$ & $\mathrm{C}$ \\
\hline $35 .^{*}$ & Crescentia cujete L. & Bignoniaceae & $\mathrm{T}$ & $\mathrm{C}$ \\
\hline $36 .^{* *}$ & Crinum amboinensis L. & Amaryllidaceae & $\mathrm{H}$ & $\mathrm{C} / \mathrm{W}$ \\
\hline $37 .^{* *}$ & Crinum powellii Baker. & Amaryllidaceae & $\mathrm{H}$ & $\mathrm{C} / \mathrm{W}$ \\
\hline $38 .^{*}$ & Crossandra nilotica Oliv. & Acanthaceae & $\mathrm{H}$ & $\mathrm{C} / \mathrm{W}$ \\
\hline $39 .^{*}$ & Cryptostegia grandiflora Roxb. & Asclepiadaceae & $\mathrm{S}$ & $\mathrm{C} / \mathrm{W}$ \\
\hline 40. & Cycas revolute $\mathrm{L}$. & Cycadaceae & $\mathrm{T}$ & $\mathrm{C}$ \\
\hline $41 *^{*}$ & Delonix regia Hook. & Caesalpiniaceae & $\mathrm{T}$ & $\mathrm{C} / \mathrm{W}$ \\
\hline
\end{tabular}

as carpet bedding, 7 species as mixed borders, 6 species as bushy and upright foliage, 3 species used in topiary formations and also, 3 species are used as ornamental palms, 2 species ornamental ferns, 15 species are recommended for railway lines 18 species are recommended for town roads, 2 species as dry wall, 3 species as topiary and 3 species as a roof gardening (Table 2-4). 


\begin{tabular}{|c|c|}
\hline $42 .^{* *}$ & Dieffenbachia picta Schott. \\
\hline $43 .^{* *}$ & Dracaena sps \\
\hline 44. & Dryopteris sps \\
\hline 45. & Duranta plumeri Jacq. \\
\hline $46 .^{* *}$ & Eichhornia crassipes Solms. \\
\hline $47 .^{*}$ & Eranthemum tricolor W.Bull \\
\hline $48 .^{*}$ & Euphoria antiquorum L. \\
\hline $49 .^{*}$ & Euphorbia heterophylla L. \\
\hline $50 .^{*}$ & Euphorbia milli Moul. \\
\hline $51 *^{*}$ & Galphimia glauca Bartl. \\
\hline $52 * *$ & Gompherena globosa L. \\
\hline $53 .^{*}$ & Heliotropium indicum L. \\
\hline $54 .^{*}$ & Hemigraphis alternate Burm.(f) \\
\hline $55 .^{*}$ & Hibiscus rosasinensis L. \\
\hline $56 .^{*}$ & Hibiscus schizopetalous L. \\
\hline $57 .^{*}$ & Hippestrum hybridum L. \\
\hline $58 .^{*}$ & Ixora coccinea L. \\
\hline $59 .^{*}$ & Jacaranda mimosifolia D.Don \\
\hline $60 *$ & Jasmium sambac L. \\
\hline $61 *^{*}$ & Jatropa hastate Jacq. \\
\hline $62 .^{* *}$ & Kalanchoebioss feldiana L. \\
\hline $63 .^{*}$ & Kleinia grandiflora L. \\
\hline $64 .^{*}$ & Lantana camera L. \\
\hline $65 .^{*}$ & Lawso niainermisL. \\
\hline 66. $\triangle$ & Microsorum pustulatum Coper. \\
\hline $67 .^{*}$ & Mirabillis jalaba L. \\
\hline $68 .^{* *}$ & Mamilaria baumii L. \\
\hline $69 .^{*}$ & Morinda coriea L. \\
\hline $70 *$ & Muntingia calabura L. \\
\hline 71.* & Mussaenda frondosa L. \\
\hline 72.* & Nymphae sps \\
\hline 73.* & Opuntia dillenii $\mathrm{L}$. \\
\hline $74^{*}$ & Opuntia rhodantha Mill. \\
\hline $75 *$ & Orthosiphon spiralis Lour. \\
\hline 76. & Oxalis corniculata $\mathrm{L}$. \\
\hline 77. $*$ & Passiflora foetida L. \\
\hline $78 .^{*}$ & Peltophorum pterocarpum Roxb. \\
\hline 79.** & Phoenix sps \\
\hline $80 .^{* *}$ & Pistia stratiotes L. \\
\hline $81 *^{*}$ & Plumeriarubra L. \\
\hline $82 .^{*}$ & Podranea bryceiL. \\
\hline 83. $*$ & Polyalthia longifolia L. \\
\hline $84 .^{* *}$ & Polyscias bulfourana Andre. \\
\hline $85 .^{*}$ & Quiqualis indica L. \\
\hline $86 .^{*}$ & Pongamia pinnata $\mathrm{L}$. \\
\hline 87. $* *$ & Rhoeo spathacea (sw) \\
\hline $88 .^{*}$ & Ruelia tuberosa L. \\
\hline 89.* & Rueliat weediana Griseb. \\
\hline $90 .^{*}$ & Russeli aequisetiformis $\mathrm{L}$. \\
\hline 91. $\bigwedge$ & Salvinia molesta $\mathrm{L}$. \\
\hline $92 .^{* *}$ & Sanseveria trifurcate L. \\
\hline $93 .^{* *}$ & Sanseveria roxburgiana Schult. \\
\hline $94 .^{*}$ & Saraca asoka Roxb. \\
\hline 95. $\triangle$ & Selaginella sps \\
\hline
\end{tabular}

\begin{tabular}{|c|c|c|}
\hline Araceae & S & $\mathrm{C} / \mathrm{W}$ \\
\hline Liliaceae & S & $\mathrm{C}$ \\
\hline Dryopteridaceae & $\mathrm{H}$ & $\mathrm{C}$ \\
\hline Verbenaceae & S & $\mathrm{C}$ \\
\hline Pontederiaceae & $\mathrm{H}$ & $\mathrm{W}$ \\
\hline Acanthaceae & S & $\mathrm{C}$ \\
\hline Euphorbiaceae & $\mathrm{Su}$ & $\mathrm{C}$ \\
\hline Euphorbiaceae & $\mathrm{H}$ & $\mathrm{W}$ \\
\hline Euphorbiaceae & $\mathrm{H}$ & $\mathrm{C}$ \\
\hline Malphiginaceae & CL & $\mathrm{C}$ \\
\hline Amaranthaceae & $\mathrm{H}$ & W \\
\hline Boraginaceae & $\mathrm{H}$ & W \\
\hline Acanthaceae & $\mathrm{H}$ & $\mathrm{C}$ \\
\hline Malvaceae & S & $\mathrm{C} / \mathrm{W}$ \\
\hline Malvaceae & S & $\mathrm{C}$ \\
\hline Acanthaceae & $\mathrm{H}$ & $\mathrm{C}$ \\
\hline Rubiaceae & $\mathrm{H}$ & $\mathrm{C}$ \\
\hline Mimosaceae & $\mathrm{T}$ & W \\
\hline Oleaceae & $\mathrm{H}$ & $\mathrm{C}$ \\
\hline Acanthaceae & S & $\mathrm{C}$ \\
\hline Crassulaceae & $\mathrm{H}$ & $\mathrm{C} / \mathrm{W}$ \\
\hline Asteraceae & $\mathrm{H}$ & $\mathrm{C}$ \\
\hline Verbenaceae & $\mathrm{H}$ & $\mathrm{C} / \mathrm{W}$ \\
\hline Lythraceae & S & $\mathrm{C} / \mathrm{W}$ \\
\hline Polypodiaceae & $\mathrm{H}$ & $\mathrm{C}$ \\
\hline Nyctaginaceae & $\mathrm{H}$ & $\mathrm{C}$ \\
\hline Cactaceae & $\mathrm{Su}$ & $\mathrm{C}$ \\
\hline Rubiaceae & $\mathrm{T}$ & W \\
\hline Tiliaceae & $\mathrm{T}$ & $\mathrm{C}$ \\
\hline Rubiaceae & S & $\mathrm{C}$ \\
\hline Nymphaeaceae & $\mathrm{H}$ & $\mathrm{C}$ \\
\hline Opuntiaceae & $\mathrm{Su}$ & $\mathrm{C}$ \\
\hline Opuntiaceae & $\mathrm{Su}$ & $\mathrm{C}$ \\
\hline Lamiaceae & $\mathrm{H}$ & $\mathrm{C}$ \\
\hline Oxalidaceae & $\mathrm{H}$ & W \\
\hline Passifloraceae & CL & W \\
\hline Fabaceae & $\mathrm{T}$ & $\mathrm{W}$ \\
\hline Palmae & $\mathrm{T}$ & $\mathrm{C}$ \\
\hline Araceae & S & $\mathrm{C}$ \\
\hline Apocynaceae & S & $\mathrm{C}$ \\
\hline Bignoniaceae & $\mathrm{H}$ & $\mathrm{C}$ \\
\hline Annonaceae & $\mathrm{T}$ & $\mathrm{C}$ \\
\hline Araliaceae & $\mathrm{H}$ & $\mathrm{C}$ \\
\hline Combretaceae & S & $\mathrm{C}$ \\
\hline Fabaceae & $\mathrm{T}$ & $\mathrm{W}$ \\
\hline Commelinaceae & $\mathrm{H}$ & $\mathrm{C}$ \\
\hline Acanthaceae & $\mathrm{H}$ & $\mathrm{W}$ \\
\hline Acanthaceae & $\mathrm{H}$ & $\mathrm{C}$ \\
\hline Scorphulariaceae & $\mathrm{H}$ & $\mathrm{C}$ \\
\hline Salvinaceae & $\mathrm{H}$ & $\mathrm{C}$ \\
\hline Convolvulaceae & $\mathrm{H}$ & $\mathrm{C}$ \\
\hline Convolvulaceae & $\mathrm{Su}$ & $\mathrm{C}$ \\
\hline Fabaceae & $\mathrm{T}$ & $\mathrm{C} / \mathrm{W}$ \\
\hline Selaginellaceae & $\mathrm{H}$ & $\mathrm{C}$ \\
\hline
\end{tabular}




\begin{tabular}{|c|c|c|c|c|}
\hline 96.* & Stylosanthe shamata L. & Fabaceae & $\mathrm{T}$ & W \\
\hline 97.** & Setcreasea purpurea Boom. & Commelinaceae & $\mathrm{H}$ & $\mathrm{C}$ \\
\hline 98.* & Swienteni amahagoni L. & Meliaceae & $\mathrm{T}$ & W \\
\hline 99.* & Santalum album L. & Santalaceae & $\mathrm{T}$ & $\mathrm{C}$ \\
\hline 100.* & Tectona grandis L.F & Verbenaceae & $\mathrm{S}$ & $\mathrm{C} / \mathrm{W}$ \\
\hline 101.* & Tecoma stans $\mathrm{L}$. & Bignoniaceae & $\mathrm{S}$ & $\mathrm{C}$ \\
\hline 102.* & Tecomaria capensis Thumb. & Bignoniaceae & $\mathrm{S}$ & $\mathrm{C}$ \\
\hline 103.* & Terminalia catappa $\mathrm{L}$. & Combretaceae & $\mathrm{T}$ & $\mathrm{C}$ \\
\hline $104 .^{*}$ & Thespesia populnea $\mathrm{L}$. & Malvaceae & $\mathrm{T}$ & W \\
\hline $105 . *$ & Thunber giaerecta Benth. & Acanthaceae & $\mathrm{H}$ & $\mathrm{C}$ \\
\hline 106.* & Tabernaemontana coronaria Schut. & Apocynaceae & $\mathrm{S}$ & $\mathrm{C}$ \\
\hline 107.* & Trapanatans L. & Trapaceae & $\mathrm{H}$ & W \\
\hline 108.* & Tridax procumbens $\mathrm{L}$. & Asteraceae & $\mathrm{H}$ & W \\
\hline
\end{tabular}

$\mathrm{H}=$ Herb; ${ }^{*}$ Dicot; $\mathrm{S}=$ Shrub; ${ }^{* *}$ Monocot; $\mathrm{T}=$ Tree; $\triangle$ Pteridophytes; $\mathrm{Cl}=$ Climber;

DGymnosperms; $\mathrm{W}=$ Wild; $\mathrm{C}=$ Cultivated; $\mathrm{Su}=$ Succulent

Table 2. Cotyledons wise distribution

\begin{tabular}{llcc}
\hline Sl. No & Nature of the plant & No. of Plants & \% \\
\hline 1. & Monocots & 28 & 25.9 \\
2. & Dicots & 74 & 68.5 \\
3. & Pteridophytes & 4 & 3.7 \\
4. & Gymnosperms & 2 & 1.8 \\
\hline
\end{tabular}

Table 3. Characterization of the recorded flora according to the ornamental utilization

\begin{tabular}{|c|c|c|}
\hline S.No & Ornamental Utility & Name of the plants \\
\hline 1. & Aquatic ornamentals & $\begin{array}{l}\text { Trapanatans, Salviniamolesta, Nymphaea sps, Pistia stratiotes, Eichhornia } \\
\text { crassipes.(4) }\end{array}$ \\
\hline 2. & Ornamental trees & $\begin{array}{l}\text { Azadirachta indica, Caryota urens, Araucaria sps, Cassia fistula, Cycas } \\
\text { revoluta, Casuarina equisetifolia, Callistemon citrinus, Cresentiacujete, Delonix } \\
\text { regia, Jacaranda mimosifolia, Thespesia populnea, Morinda coriea, Polyalthia } \\
\text { longifolia, Pongamia pinnata, Peltophorum pterocarpum, Plumeria rubra, } \\
\text { Muntingia calabura, Albizzia julibrissin, Albizzia lebbeck, Terminala catappa, } \\
\text { Santalum album, Swietenia mahagoni (22) }\end{array}$ \\
\hline 3. & Ornamental shrubs & $\begin{array}{l}\text { Tectona grandis, Tecomaria capensis, Tecomastans, Russeliae quisetiformis, } \\
\text { Phoenix sps, Polyscias bulfourana, Mussaenda frondosa, Lantana camera, } \\
\text { Lawsonia inermis, Jatropaha stata, Ixora coccinea, Hibiscus schizopetalous, } \\
\text { Hibiscus rosasinesis, Hippestrum hybridum, Hemigraphis alternate, } \\
\text { Eranthemum tricolor, Dracaena sps, Duranta plumerie, Dieffenbachia pictata, } \\
\text { Calotropis gigantea, Chlolrophytum cosmosum, Cordylinesps, Cassia biflora, } \\
\text { Adathoda vasica (24). }\end{array}$ \\
\hline 4. & Ornamental herbs & $\begin{array}{l}\text { Alocasia macrorhiza, Aristalochaia indica, Barleria prionitis, Balsam } \\
\text { impatiens, Begonia fiocifera, Caladium bicololur, Cathranthus roseus, } \\
\text { Commelinea benghalensis, Crinum powellii, Crinum amboinensis, Coleus } \\
\text { blumei, Coleus amboinicus, Euphorbia heterophylla, Clerodendrum speciosum, } \\
\text { Gompherena globosa, Hippestrum hybridum, Keleinia grandiflora, Mirabilis } \\
\text { jalaba, Oxalis corniculata, Stylosanthus hamatus, Rhoeospa thacea, Ruelia } \\
\text { tweediana, Ruelia tuberosa, Orthosiphon spiralis, Tridax procumbens, Setrea } \\
\text { seapurpurea, Sanseveria roxburghiana, Sanseveria trifuscata. (27) }\end{array}$ \\
\hline 5. & Ornamental Hedge & $\begin{array}{l}\text { Polyalthia longifolia, Casuarina equisetifolia,Albizzia lebbeck, Bougainvillea } \\
\text { spectabilis, Delonixregia, Callistemon citrinus, Azadiracta indica, Jacaranda } \\
\text { mimosifolia, Plumeria rubra, Pongamia pinnata, Muntingia calabura, } \\
\text { Santalam album, Thespesia populnea, Hibiscus rosasinensis, Tecomastans, } \\
\text { Tecomaria capensis. (16) }\end{array}$ \\
\hline 6. & Ornamental & Euphorbia antiquorum, Mamillaria baumii, Opuntia dilleni, Opuntia \\
\hline
\end{tabular}


succulent \& cacti rhodantha.(4)

7. Ornamental climber Allamanda carthartica, Asparagus race mosus, Cryptostegia grandiflora, Bougainvillea spectabilis, Clitoriaternatea, Cissus quadrangularis, Galphimia glauca, Jasminum sambac, Passiflora foetida, Quiqualis indica, Thunber giaerecta.(11)

8. Ornamental foliage Alocasia macrorhiza, Coleus blumei, Cordyline sps, Codiaeum variegatum, Caladium bicolour, Dieffenbachia picta, Dracenasps, Dryopteris sps, Chlorophytum cosmosum, Kleinia grandiflora, Phoenix sps, Micorosorum pustulatum, Sanseveria trifuscata, Sanseveria roxburgiana, Begonia flocifera, Asparagus racemosus, Cycasrevoluta, Setreasea purpurea, Commelina benghalensis, Anthurium sps. (20)

9. Carpet bedding Coleus blumei.(1)

10. Mixed Border

Euphorbia heterophylla, Tecomastans, Thunber giaerecta, Catharathus roseous, Crossandra nilotica, Caesalpinia pulcherrima, Tecomaria capensis. (7)

11. Trailers

Setcrea seapurpurea, Chlorophytum comosum.(2)

12. Bushy \& upright foliage

Aracucaria sps, Begonia flocifera, Cordyline sps, Dieffenbachia picta, Dracena sps, Setcreasea purpurea.(6)

13. Topiary

14. Ornamental palms

15. Ornamental ferns

16. Ornamental trees Bougainvillea spectabilis, Clerodendrum speciosum, Durantaplumerri.(3)

Cycasrevoluta, Phoenix, Caryotaurens.(3)

Microsorum pustutatum, Selaginella sps.(2) recommended for indica, Muntingia calabura, Albizzi ajulibrissin, Cassia fistula, Callistemon town roads

citrinus, Thespesia populnea, Pongamia pinnata, Jacaranda mimosifolia, Cresentia cujete, Albizzia lebbeck, Peltophoruminerne, Terminalia catappa, Santalum album, Swietenia mahagoni.(18)

17. Ornamental trees Albizzia lebbeck, Peltophorump terocarpum, Terminalia catappa, Santalum recommended for alum, Swietenia mahagoni, Delonix regia, Cassia fistula, Pongamia pinnata, Railway line. Thespesia populnea, Albizzia julibrissin, Saracaasoca, Azadiracta indica, Polyalthia longifolia, Jacaranda mimosifolia, Cresentiacujete.(15)

18. Dry wall

19. Hanging baketes Oxalis corniculata, Selaginella sps.(2)

Alocasia macrorhiza, Anthurium sps, Mamillaria baumii, Caladium bicolour, Chlorophytum comosum, Cissus qudrangularis, Commelina benghalensis, Dryopteris sps, Microsorum pustalatum (9)

20. Roof gardening Bougainvillea spectabilis, Plumeriarubra, Passiflora foetida.(3)

Table 4. Habitat wise distribution of identified plants

\begin{tabular}{ccc}
\hline Sl. No & Habitat & No. of Plants \\
\hline 1. & Climber & 4 \\
2. & Succulent & 8 \\
3. & Shrub & 26 \\
4. & Herb & 48 \\
5. & Tree & 22 \\
\hline
\end{tabular}

Landscape gardening and bio-aesthetic planning is a recent trend to establish eco-friendly human habitats. Exploration of collection and conservation of wild and cultivated ornamental species is also one of the cultural methods to maintain the diversity of the species and conserve to endemic and endangered species of ornamental interest. There is a lot of significance in recent year for the ornamental species in the utilization of various kinds and is the income generation among poor also in the export market of India. Wild ornamental species are also the sources for the medicinal significance (Asati and Yadav, 2010) so the ornamental germplasm relatives are to be conserved. In the development for new hybrids, polyploidy mutation of ornamental interest it is essential to know ornamental species. The dynamic floriculture industry is constantly looking for new products, technology and market riches. 
Over-exploitation by humans, both for direct consumption and also for botanical and horticulture value, also threatens wild ornamentals. Grassland reclamation programmes and overgrazing by cattle have had a debilitating effect on these wild species. Fragmentation of extensive habitats into small isolated patches can mean that they become too limited to maintain their plant populations. Fragmentation seems to reduce genetic variation and seedling vigour. Natural disasters have also played a role in species extinction. Even protected areas cannot be expected to safeguard plants from the effects of disasters such as volcanoes, fires, airborne pollutants, droughts and landslide.

This process is largely based on research and development and requires strong collaboration between many links of the production chain most modern scientific research in the field of new ornamental crops deals with the adaptability of new species to the environmental and the regulation of their life cycle or propagation systems. New ornamental products can be developed by researches and breeders only in collaboration with efficient produces and satisfied consumes, linked together in mutually beneficial ways. It is very easy for the propagation of wild species by traditional propagation methods. The cost of domestication and maintenanceofornamental species is also very less in comparison. We hope this work will help the researches and people who are interested in ornamental plants.

\section{REFERENCES}

Aasati, B.S. and D.S. Yadav, (2000). Diversity of horticultural crops in North Eastern region. ENVIS and Utilization of Ornamental Germplasm.

Arora, L. (1998). Shade-tolerant flowering plants in the southern African flora: Morphology, adaptations and horticultural application. M.Sc. thesis, University of Pretoria, South Africa.

Gamble, J.S., and C.E.C. Fischer, (1915-1936). Flora of the presidency of Madras. Vol.1, Adlard \& Sons Ltd., London.

Henry, A.N., V. Chithra and N.P. Balakrishnan, (1989). Flora of Tamil Nadu. Series 1, Vol.3, Botanical Survey of India, Coimbatore.

Jenomics, W., (2008).The greening of art: ecology, community and the public domain. South Afr. J. Art Hist. 23(1):175-189.

Pullaiah, K. Sri Ramamurthy and S. Karuppusamy, (2007). Eastern Ghats hill ranges of south east. 